\title{
Framework of Electrical Fire Probability Estimation Based on Bayesian Network Model Inference
}

\author{
Guohua WU,b,c Xiaoqing CHEN ${ }^{a}$, Jiyao YIN ${ }^{a}$, Diping YUAN ${ }^{a}$, Yihua HU, ${ }^{\text {a,d }}$, Yiping \\ $\mathrm{ZENG}^{\mathrm{e}, 1^{*}}$, Kang $\mathrm{LIU}^{\mathrm{f}}$ and Xin $\mathrm{ZHAO}^{\mathrm{a}}$ \\ a Shenzhen Urban Public Safety and Technology Institute, Shenzhen 518000, China \\ ${ }^{\mathrm{b}}$ Harbin Institute of Technology, Shenzhen 518000, China \\ c Shenzhen Urban Transport Planning Center Co. Ltd, Shenzhen 518000, China \\ ${ }^{\mathrm{d}}$ Department of Aerospace, Mechanical and Mechatronic Engineering, University of \\ Sydney, Faculty of Engineering, 456P+HW Camperdown, New South Wales, Australia \\ e Southern University of Science and Technology, Shenzhen 518000, China \\ f Shenzhen Institute of Advanced Technology, Chinese Academy of Sciences, Shenzhen \\ 518000, China
}

\begin{abstract}
Electrical fire had become one of the main parts in total fire accidents. Most of researches rely on the complex combustion models, which consume a huge number of computational resources. However, few studies focus on evaluating fire disaster by probability theory, and estimate the likelihood of fire occurring by the calculation result of probability based on the current data from the sensor. Bayesian Network is introduced due to the advantage of calculation complexity, ability of expressing uncertain factors and the accuracy of model with incomplete data. Some problems should be solved before using Bayesian Network to inference events based on given evidences. In this paper, the structure and the parameter of the Bayesian Network is created by the discussing result of the experts and scholars in electrical fire research field. A frequently-used fuzzy function called Sigmoid function to process data from raw data to the probability. Inference result by Bayesian Network is calculated by the Variable Elimination algorithm. A case study about the simulation of analyzing the probability of electrical fire happened when the load of circuit is under the high status. Research result shows that Bayesian Network model is suitable for estimating and analyzing in the scenario of electrical fire. Model has a good robust to express probability of electrical fire probability, which is of vital importance for estimating whether the fire occurs or not, thus providing significant information and instruction for preventing electrical fire and the sustainability of the environment. Based on the simulation result, it can conclude that the Bayesian network model inference is suitable for the electrical fire estimation scenario, and the introducing of this scheme is possible for predict electrical fire.
\end{abstract}

Keywords. Electrical fire, Bayesian Network, variable elimination algorithm

${ }^{1}$ Corresponding Author, Yiping ZENG, Southern University of Science and Technology, Shenzhen 518000, China; Email: 1926397162@qq.com. 


\section{Introduction}

Electrical fire had become one of the main parts in total fire accidents. According to the statistics result, from 1993 to 2007, about 373700 electrical fires occurred all around the world. It occupies $24.5 \%$ of the total fire accidents on average. From 1997 to 2007, 1324 catastrophic electrical fire accidents occurred nationwide. They accounted for $31.5 \%$ and $44.3 \%$ of all major fires respectively [1].

Besides, when the fire is happening, interior materials will be resolved, with producing carbon dioxide, harmful gas emissions ( $\mathrm{Such}$ as $\mathrm{HCl}, \mathrm{HCN}$ and $\mathrm{CO}$ ) for the indoor fire, and $\mathrm{CO}, \mathrm{CO}_{2}, \mathrm{NO}, \mathrm{NO}_{2}$, hydrocarbons, organic carbon, and inorganic elements, and PM2.5 for the wildland fire and forest fire [2,3], which not only pose a threat to the health of people, but also bring negative influences to the environment. It is necessary to reduce occurring times of electrical fire for the sustainability of environment.

Current researches mainly focus on estimating fire in several ways. Kou et al. introduced an estimation method based on deep learning and data mining to learn data and evaluate models [4]. Angelo A et al. used level set method to construct fire propagation model for estimating the speed and direction of fire propagation, which can be used to instruct how to estimate and put on the fire [5]. Kim et al. implement a rotational UV sensor to detect the position of the fire resource, and calculate the accuracy of fire location bases on Bayesian estimation [6]. For the forest fire, a UAV is used to collect information about wind and other factors (such as humidity, vegetation coverage) to estimate wind flow for calculating the midflame windspeed of the forest fire [7]. A method about the combination of data assimilation and fire propagation model is used to provide the accurate estimation for the fire propagation and forecasting fire [8].

The paper is following by the above structure to prove the possibility of this approach, the flowchart of working steps of this paper is shown in Figure 1. Firstly, an expert-based Bayesian Network structure and conditional probability tables are constructed and then used to inference the probability of electrical fire. Secondly, sensor data is processed by the Sigmoid function. Thirdly, processed probability is the evidence of the Bayesian Network inference, and inference the result by variable elimination algorithm. Simulation is used to evaluate the robust and accuracy of the Bayesian Network inference under two cases, complete data and missing data respectively.

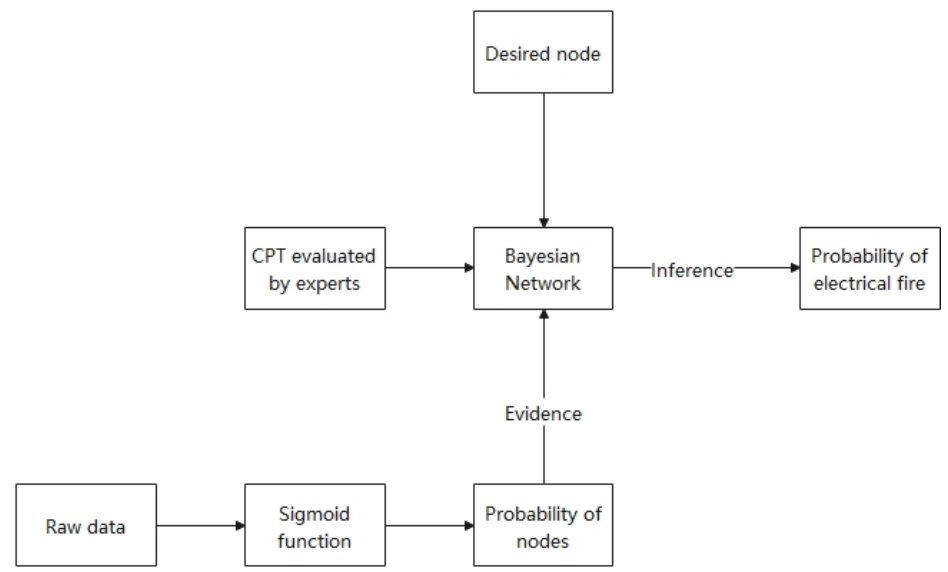

Figure 1. Framework of fire probability inference by Bayesian Network. 


\section{Sigmoid Function}

In this paper, the parameters of nodes only contain 2 possible results, which includes " 0 " and " 1 ". We can consider that the following formula is satisfied in this situation.

$$
P(x=0)+P(x=1)=1
$$

The Sigmoid membership function is used to evaluate the probability of uncertain events, and this function is suitable to evaluate events with 2 statuses by using probability. The formula of the Sigmoid function is shown as

$$
f(x, k, c)=\frac{1}{1+e^{-k(x-c)}}
$$

Figure 2 presents the Sigmoid function curve for the "current" factor, the trend of the function is like a S-curve, the parameters of the current Sigmoid function are $\mathrm{k}=20$, $\mathrm{c}=0.8$ respectively.

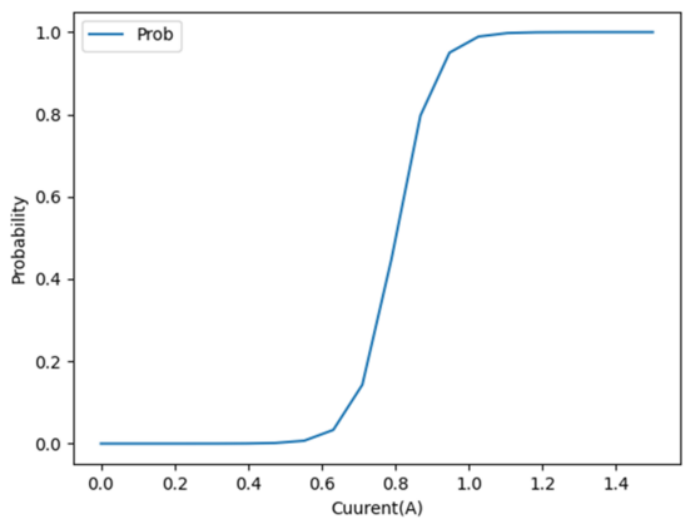

Figure 2. Sigmoid function of the "current" factor.

In this case, current value acts as the input of the Sigmoid function, and the output result represents the probability that "Current" events is under the "1" status, which means high current value. The probability of " 0 " status is equal to the difference between 1 and the probability of " 1 " status. For example, when the temperature sensor shows the current value is $0.8 \mathrm{~A}$ at moment, the convert result of Sigmoid function is $(0.5,0,5)$, which means that the probability of "high current status" and "not a high current status" have the same probability value. We consider that this current value $(0.8 \mathrm{~A}$, the same as parameter $\mathrm{c}$ in the Sigmoid function formula) is the threshold of the state transition.

\section{Bayesian Network}

\subsection{Principle of Bayesian Network}

The Bayesian belief network, known as the $\mathrm{BN}$, is a directed acyclic graph that consists 
of nodes and directed edges. The system's parameters are represented by nodes. The node state generally represents the state of continuously updated parameters, such as temperature and pressure, which are evaluated initially with Sigmoid Function. Figure 3 illustrates the topology of simplify Bayesian Network.

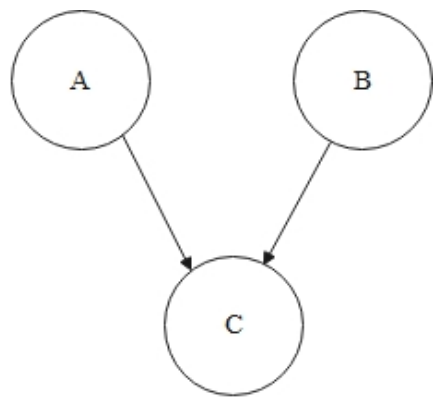

Figure 3. Example of Bayesian Network.

\subsection{Bayesian Inference}

Bayesian network inference is a process to obtain the state information of unknown nodes based on the existing evidence information. According to the direction of network inference, it can be divided into three kinds of inference: forward inference, reverse inference and explanatory inference $[9,10]$. Positive inference is to know the cause of occurrence and infer all possible consequences; Reverse inference is to analyze the possible causes of the phenomenon according to the phenomena and consequences that have occurred.

\subsection{Probability Calculation Based on Bayesian Network Inference}

In order to estimate the probability of desire events, the following steps have been followed.

Step 1: Discuss the possible factors in the applied scenario with experts, and ensure the structure of Bayesian Network model based on expert's professional experience.

Step 2: Build CPTs for each node in Bayesian Network model, the data of conditional probability about each factor is evaluated by the expert.

Step 3: Sigmoid Function is carried out to convert raw information from the sensor to the probability of the node. In the given example in section 2. Current data is converted to the probability of current status, from $0.8 \mathrm{~A}$ to $(0.5,0.5)$.

Step 4: Ensure the evidence of the Bayesian Network, for the example in section 2, the current evidence in this moment is $(0,1)$, which means that status of current node is "High".

Step 5: Inference Bayesian Network model with the variable elimination algorithm, return the output probability of the model. In the electrical fire case, when the current evidence is $(0,1)$, the probability of occurring electrical fire is 0.5133 based on the variable elimination.

Step 6: Analyze the output of Bayesian Network in two situations, one is the scenario with complete data, and the other is the case with the lack of partial raw data from the sensors. 


\section{Case Study: Electrical Fire Analysis Based on Bayesian Network Inference}

\subsection{Bayesian Network Model}

\subsubsection{Bayesian Network Model for Electrical Fire}

A flowchart of the Bayesian Network model is present in Figure 4.

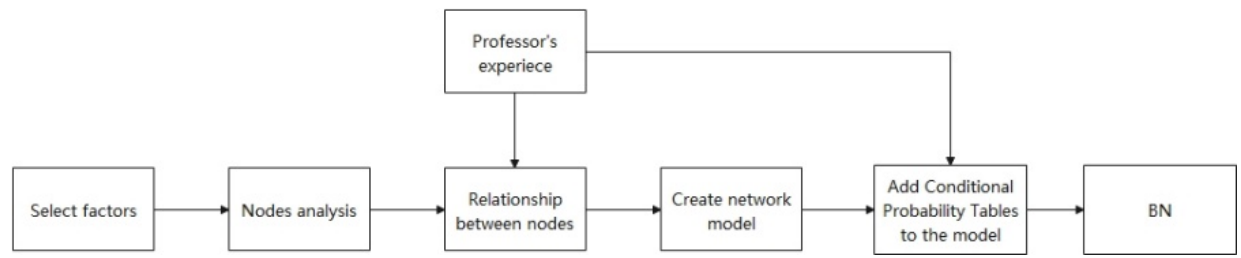

Figure 4. Process of constructing Bayesian Network.

In this case study, we choose several nodes to analyze the phenomenon between the nodes and the status when the raw data of node is changed. Take the "current" node as an example, when the indoor sensor detects that the circuit current is rising rapidly, the load of the circuit will rise, and the circuit temperature will rise at the same time because of the Joule's law.

When all of the nodes are set up to the model, and the CPTs are finished by adding probability from the professors, the Bayesian network modelling is finished, and it can be the inference model in the next section. Figure 5 illustrates the structure of the Bayesian network, which represents the relationship between factors.

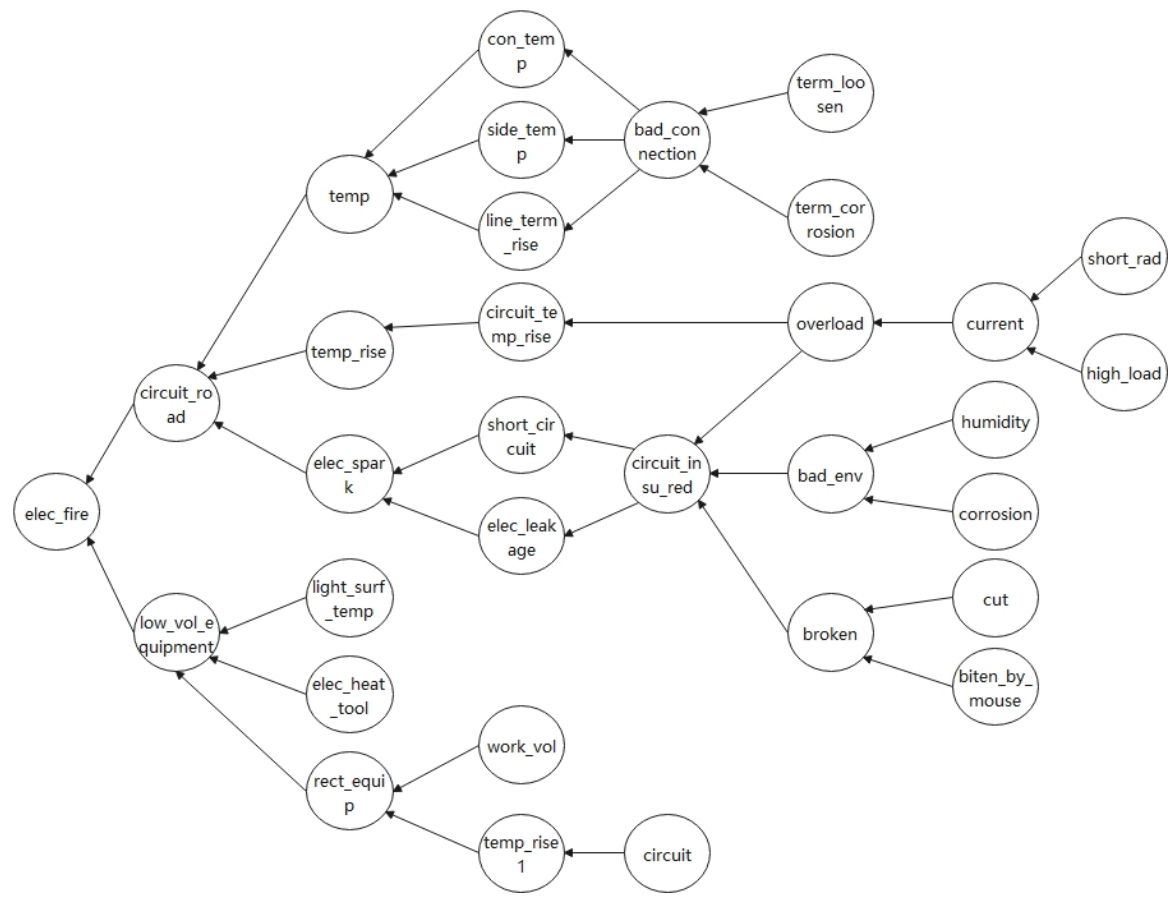

Figure 5. Structure of Bayesian Network for the electrical fire probability calculation. 


\subsubsection{Relationship between Data}

To ensure the correctness of the Bayesian network model, a simulation is needed to show the changing trends of raw data when the parameter is changing. Figure 6 and Figure 7 show the test result of the simulation when the circuit load is high at $5 \mathrm{~min}$, and proves the accuracy of the Bayesian Network.

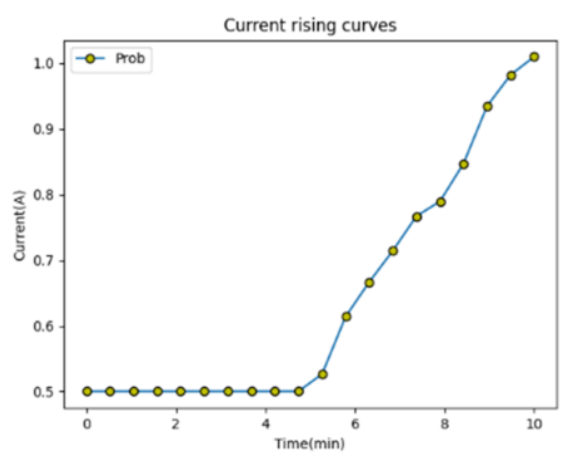

Figure 6. Current rising curve when the high load is true.

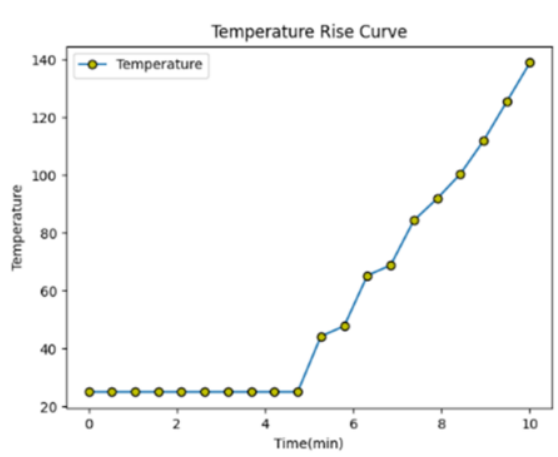

Figure 7. Temperature rising curve.

\subsection{Information Processing}

Figure 8 is the sigmoid function images for the "temp" node. In this figure, the parameter of Sigmoid function is set as $\mathrm{k}=1 / 3, \mathrm{c}=25$.

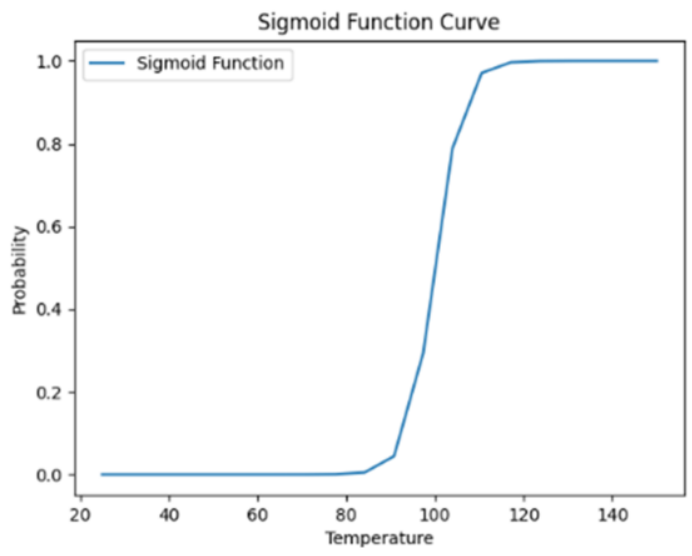

Figure 8. Sigmoid function of the "temperature" factor.

\subsection{Electrical Fire Probability Analysis Based on Bayesian Network Inference}

\subsubsection{Inference with No Sensor Broken}

Assume that at 5th minute, circuit load becomes high, which means that temperature starts to rise at the same time. The probability of "High temperature" is shown in Figure 9. When temperature is closing to the temperature default threshold, the probability of high temperature status rises dramatically. With the increasing temperature, the high temperature status is becoming a convincing factor when the probability is bigger than 0.5 . 


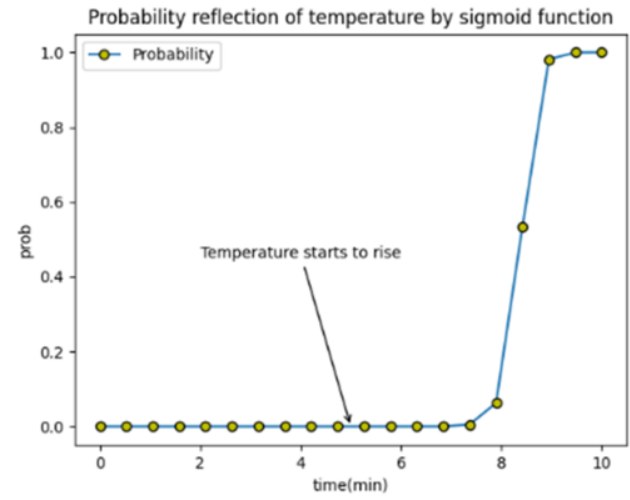

Figure 9. Probability of temperature in "High" status.

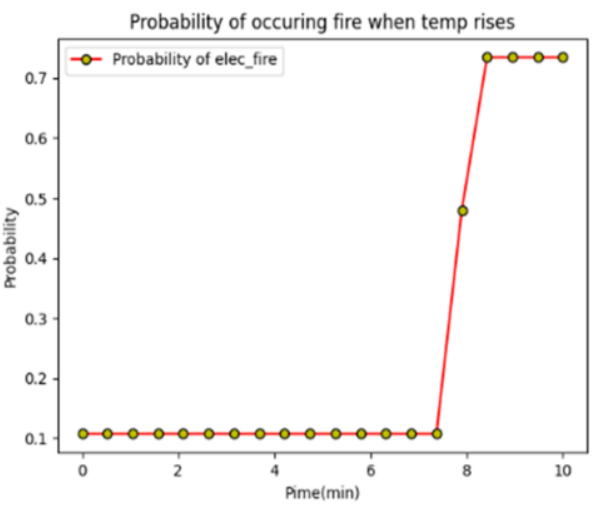

Figure 10. Probability of electrical fire occurs.

Bayesian Network is used to inference the probability of electrical fire during the 10 minutes period, since the temperature sensor is well working. The evidences of the Bayesian include the status of the "high_load", "temp" and "current", and the probability of occurring fire trend is shown in Figure 10. The higher the probability, the higher the likelihood that the electric fire happened.

\subsubsection{Inference with Temperature Sensor Broken}

Assume that temperature sensor has been failed all the time during the time period 0-10 minute. In this case, the evidence of "temp" node is missed. Bayesian Network inference result with incomplete data is presented in Figure 11. Bayesian Network can inference the result of electrical fire probability in a high performance, but the data is lower authoritative than the complete data inference result.

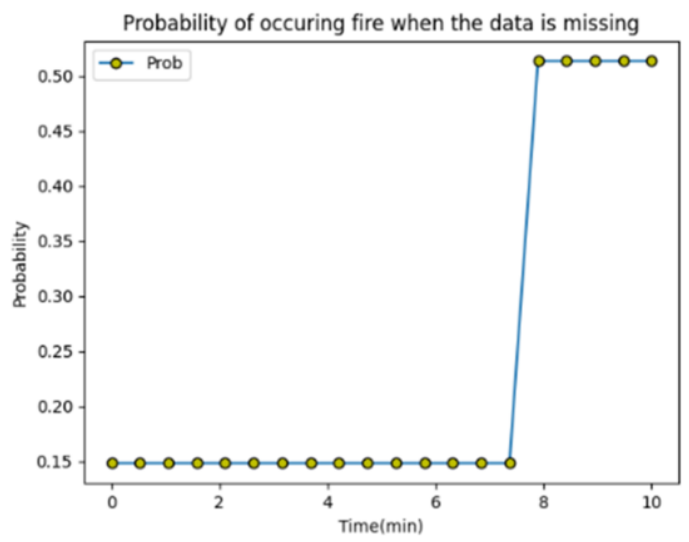

Figure 11. Probability of electrical fire occurs when the temperature sensor data is missing.

Bayesian Network provides reliable result for detecting the electric fire when the temperature is rising. Although the raw data of the inference evidence is lacking, it still has higher accuracy to estimate the probability of electric fire occurred with the inference of "high_load" and "current" nodes data. The simulation result shows that the accuracy 
of the inference is higher enough to estimate when the electrical happens in a high probability, however, the more information loss for the Bayesian Network, the less accuracy for inferencing result from the Bayesian Network. The outcome of the paper can be used to deal with real cases in engineering field, an electrical fire estimation system can be designed based on the framework of the electrical fire analysis by using Bayesian Network inference.

\section{Conclusion}

A method to estimate the probability of electrical fire was introduced. Firstly, the Bayesian Network structure and conditional probability tables are provided by the experts in safety engineering field. Secondly, the original data from sensors are processed by Sigmoid function, which is able to convert raw data to the probability distribution of the node. Thirdly, the likelihood of happening electrical fire can be calculated by providing sensor data as the inference evidence, by using variable elimination method. Finally, in order to prove the possibility of electrical fire estimation, the simulation of the circuit high load and the missing data of temperature sensor are tested. Simulation result shows that Bayesian Network model is able to perform the probability of happening electrical fire, and model can inference the electrical fire result with the lack of information status.

However, electrical fire is a complex scenario, with a huge number of uncertain factors and the large difference between the occurring places. Although this paper raises a framework of realizing the electrical fire prediction and warning method, using this framework in general situation is a challenging issue. Besides, this framework strongly relies on artificial information. Expert's instruction will affect the accuracy and performance of the Bayesian Network. In the future, we will focus on using machine learning to build CPTs automatically for reducing human disturbance. The multiple evidence information missing for the Bayesian Network is also our research target in the next step.

\section{Acknowledgements}

This research was supported by National Key R\&D Program of China (2020YFB2103705 \& 2019YFC0810705) and Shenzhen Postdoctoral Research Fund (Grant Number: K21627501).

\section{References}

[1] Gao D and Liu Q 2015 Review of the research on the identification of electrical fire trace evidence 2015 7th International Conference on Performance-Based Fire and Fire Protection Engineering pp 29-32.

[2] Guo L, Ma Y, Tigabu M, Guo X, Zheng W and Guo F 2020 Emission of atmospheric pollutants during forest fire in boreal region of China Environmental Pollution 264.

[3] Aurell J, et al. Forest, Utah using an unmanned aircraft system, 2021 Wildland fire emission sampling at Fishlake National Atmospheric Environment 247.

[4] Kou L, Wang X, Guo X, Zhu J and Zhang H 2021 Deep learning-based inverse model for building fire source location and intensity estimation Fire Safety Journal 121103310. 
[5] Alessandri A, Bagnerini P, Gaggero M and Mantelli L 2021 Parameter estimation of fire propagation models using level set methods, Applied Mathematical Modelling 92 731-747.

[6] Kim J H and Moon S 2020 Recursive Bayesian estimation based indoor fire location by fusing rotary UV sensors 2020 IEEE/ASME International Conference on Advanced Intelligent Mechatronics pp 528-533.

[7] Xing Z, Zhang Y, Su C, Qu Y and Yu Z 2019 Kalman filter-based wind estimation for forest fire monitoring with a quadrotor UAV 2019 IEEE Conference on Control Technology and Applications pp 783-788.

[8] Zhang C, Collin A, Moireau P, Trouvé A and Rochoux M C 2019 State-parameter estimation approach for data-driven wildland fire spread modeling: Application to the 2012 RxCADRE S5 field-scale experiment Fire Safety Journal 105 286-299.

[9] Cai B, Liu Y, Liu Z, Tian X, Zhang Y and Ji R 2013 Application of Bayesian networks in quantitative risk assessment of subsea blowout preventer operations Risk Analysis 33 1293-1311.

[10] Perez-Solano J J and Felici-Castell S 2017 Improving time synchronization in wireless sensor networks using Bayesian inference Journal of Network and Computer Applications 82 47-55. 